\title{
Bacteriophage Therapy Rescues Mice Bacteremic from a Clinical Isolate of Vancomycin-Resistant Enterococcus faecium
}

\author{
Biswajit Biswas, ${ }^{1,2}$ Sankar Adhya, ${ }^{1}$ Paul Washart, ${ }^{1,2}$ Brian Paul, ${ }^{1}$ Andrei N. Trostel, ${ }^{2}$ \\ Bradford Powell, ${ }^{1,2}$ Richard Carlton, ${ }^{2}$ and Carl R. Merril ${ }^{1 *}$ \\ National Institutes of Health, Bethesda, Maryland 20892, ${ }^{1}$ and Exponential Biotherapies, Inc., \\ Pt. Washington, New York $11050^{2}$
}

Received 30 August 2001/Returned for modification 26 September 2001/Accepted 18 October 2001

\begin{abstract}
Colonization of the gastrointestinal tract with vancomycin-resistant Enterococcus faecium (VRE) has become endemic in many hospitals and nursing homes in the United States. Such colonization predisposes the individual to VRE bacteremia and/or endocarditis, and immunocompromised patients are at particular risk for these conditions. The emergence of antibiotic-resistant bacterial strains requires the exploration of alternative antibacterial therapies, which led our group to study the ability of bacterial viruses (bacteriophages, or phages) to rescue mice with VRE bacteremia. The phage strain used in this study has lytic activity against a wide range of clinical isolates of VRE. One of these VRE strains was used to induce bacteremia in mice by intraperitoneal (i.p.) injection of $10^{9} \mathrm{CFU}$. The resulting bacteremia was fatal within $48 \mathrm{~h}$. A single i.p. injection of $3 \times 10^{8} \mathrm{PFU}$ of the phage strain, administered 45 min after the bacterial challenge, was sufficient to rescue $100 \%$ of the animals. Even when treatment was delayed to the point where all animals were moribund, approximately $50 \%$ of them were rescued by a single injection of this phage preparation. The ability of this phage to rescue bacteremic mice was demonstrated to be due to the functional capabilities of the phage and not to a nonspecific immune effect. The rescue of bacteremic mice could be effected only by phage strains able to grow in vitro on the bacterial host used to infect the animals, and when such strains are heat inactivated they lose their ability to rescue the infected mice.
\end{abstract}

Isolates of vancomycin-resistant Enterococcus faecium (VRE) from patients in the United States, France, and England were first reported in 1989 (9, 19). By 1998, the U.S. National Nosocomial Infections Surveillance System had reported that $20 \%$ of nosocomial isolates of enterococci were resistant to vancomycin (12). Individuals with compromised immune systems, such as AIDS patients, cancer patients undergoing chemotherapy, postsurgical patients, transplant recipients, and the elderly in general, are particularly prone to develop VRE infections. While the antibiotic quinupristindalfopristin (Synercid; Rhone-Poulenc Rorer, Collegeville, Pa.) has recently been licensed for clinical use, its efficacy for VRE infections may be limited because (i) it is bacteriostatic, and (ii) one of its two components is an analog of virginiamycin, which has been used as an additive in hog and poultry feed for the past 2 decades. Quinupristin-dalfopristin-resistant bacteria have been isolated from turkeys fed virginiamycin, suggesting that the use of virginiamycin has created a reservoir of enterococci resistant to the analog in quinupristin-dalfopristin (5). Linezolid (Zyvox; Pharmacia and Upjohn), another recently introduced antibiotic, is also described as bacteriostatic for VRE, and resistance to it appeared during clinical trials even though it is the first member of a new class of agents (the oxazolodinones).

Early applications of antibacterial phage therapy (1920s to 1950 s) were impeded by a number of factors. One factor was the use of phage strains whose host range was too narrow, a

\footnotetext{
* Corresponding author. Mailing address: NIMH/NIH, 9000 Rockville Pike, Bldg 10, Room 2D54, Bethesda, MD 20892. Phone: (301) 435-3583. Fax: (301) 480-9862. E-mail: merrilc@helix.nih.gov.
}

problem we have largely overcome by selecting enterococcal phages able to infect the vast majority of clinical isolates of VRE. Another factor was the large load of endo- and exotoxins in the bacterial debris present in the filter-sterilized but otherwise unpurified phage lysates that past investigators administered parenterally as well as orally, a problem we have overcome with cesium chloride density centrifugation and other modern techniques of phage purification. A third factor was sterilizing phage preparations by heat and/or by the addition of mercurials and oxidizing agents, procedures that are now known to inactivate phages and that we avoid. Finally, the pharmacokinetics of phage therapy were essentially ignored in clinical applications of phage therapy, the assumption being that oral and parenteral administration would achieve concentrations at the sites of infection sufficient to induce a cure. A pharmacokinetic study performed in 1973 demonstrated the rapid clearance of phage from the bloodstream and accumulation of phage particles in the spleen and other filtering organs of the reticuloendothelial system, where they remain viable for at least a week (7). This passive capture by and sequestration in the filtering organs would prevent the vast bulk of administered phage particles from reaching the infecting bacteria, and it would add variability in therapeutic applications. To address this problem, serial passage methods have been developed to select and enrich for phage strains whose rate of clearance by the reticuloendothelial system is reduced. The long-circulating phage strains produced have been shown to be superior to the wild types from which they were derived for rescuing animals from otherwise fatal bacteremias due to Escherichia coli and Salmonella enterica serovar Typhimurium (11). Following these studies, we chose to work with VRE 
because it is a clinically important, multidrug-resistant pathogen. In the present study we report the isolation of bacteriophage that are safe and effective as bactericidal agents for animals with lethal VRE infections. The results warrant a reexamination of the potential application of bacteriophages in Western medicine.

\section{MATERIALS AND METHODS}

Isolation and purification of phage strains. The strains of E. faecium used in this study were a VRE strain isolated from a patient's blood, designated CRMEN 44 in our nomenclature and (ii) vancomycin-susceptible E. faecium (VSE) strain ATCC 19950 from the American Type Culture Collection. A strain of Enterococcus faecalis was isolated from a patient's fecal material and is designated CRMEN 19 in our nomenclature. The Enterococcus phages ENB6 and C33 were isolated from raw sewage at a municipal sewage treatment plant. The isolations, performed similarly but individually for each phage strain, were accomplished by adding salt $(58 \mathrm{~g}$ of $\mathrm{NaCl})$ to 1 liter of sewage followed by centrifugation at $10,000 \times g$ for $10 \mathrm{~min}$. The supernatant was decanted into a separate container and mixed with polyethylene glycol (PEG; molecular weight, $8,000)$ to provide a final concentration of PEG of $10 \%(\mathrm{wt} / \mathrm{vol})$. The PEGcontaining supernatant was precipitated overnight at $4{ }^{\circ} \mathrm{C}$ and centrifuged at $12,000 \times g$ for $20 \mathrm{~min}$. The resulting precipitate was dissolved in $5 \mathrm{ml}$ of phage dilution buffer (SM) and extracted once with an equal volume of chloroform. An aliquot $(300 \mu \mathrm{l})$ of this processed sewage was mixed with $200 \mu \mathrm{l}$ of an overnight culture of E. faecium ATCC 19950 , incubated at $37^{\circ} \mathrm{C}$ for $20 \mathrm{~min}$, mixed with 2.5 $\mathrm{ml}$ of molten top agar $(0.6 \%$ agar $)$ at $50^{\circ} \mathrm{C}$, and poured on Streptococcus faecalis agar plates $\left(1.5 \% \mathrm{SF}\right.$ agar; see below). Plates were incubated overnight at $37^{\circ} \mathrm{C}$. Phage plaques were harvested from the plate and single plaques were purified three times on E. faecium ATCC 19950 by the standard procedures described by Sambrook et al. (14).

Phage ENB6, used in animal rescue experiments, was grown in a CRMEN 39 host to make high-titer stocks by standard procedures (11). Large-scale preparations and purifications of bacteriophage by cesium chloride density centrifugation were performed as described by Sambrook et al. (14). Toxin levels in phage preparations were measured by the Limulus amebocyte lysate assay (Associates of Cape Cod, Inc., Woods Hole, Mass.). High-titer phage preparations were filtered through a $0.22-\mu \mathrm{m}$ filter (GS filter; Millex, Millipore Corp., Bedford, Mass.) prior to animal inoculation. These filtrates, containing high-titer phage, were stored at $4^{\circ} \mathrm{C}$. The phage strain $\mathrm{C} 33$ was grown and purified in a similar manner for use in animal experiments.

Establishing the MLD in the mouse model. One-month-old BALB/c female mice (in groups of five, except where otherwise noted) were used for infection experiments. Preparation of the infecting bacteria was as follows. VRE strain CRMEN 44 was grown in SF medium (20 g of pancreatic digest of casein, $5 \mathrm{~g}$ of glucose, $5 \mathrm{~g}$ of NaCl, $4 \mathrm{~g}$ of $\mathrm{K}_{2} \mathrm{HPO}_{4}$, and $1.5 \mathrm{~g}$ of $\mathrm{KH}_{2} \mathrm{PO}_{4}$ in 1 liter of $\mathrm{H}_{2} \mathrm{O}$, with the $\mathrm{pH}$ adjusted to 6.1 ) at $37^{\circ} \mathrm{C}$. $\mathrm{Log}$-phase cultures of CRMEN 44 were grown to an optical density at $600 \mathrm{~nm}$ of 1.1 (equivalent to $5 \times 10^{8} \mathrm{CFU} / \mathrm{ml}$ ), followed by centrifugation and resuspension in sterile phosphate-buffered saline (PBS) at $4^{\circ} \mathrm{C}$. This log-phase preparation of bacteria was serially diluted in PBS for use in animal infections. To determine the minimum lethal dose (MLD), serial dilutions of CRMEN 44 were injected intrperitoneally (i.p.) into mice in $400-\mu \mathrm{l}$ aliquots. The animals were observed for $100 \mathrm{~h}$. Mice inoculated with bacteria were scored for their state of health on a scale of 5 to 0 , based on progressive disease states reflected by several clinical signs. A normal and unremarkable condition was scored as 5; slight illness, defined as lethargy and ruffled fur, was scored as 4 ; moderate illness, defined as severe lethargy, ruffled fur, and hunched back, was scored as 3; severe illness, with the above signs plus exudative accumulation around partially closed eyes, was scored as 2 ; a moribund state was scored as 1 ; and death was scored as 0 . While the experiments were not conducted in a double-blind manner, all animals were evaluated by two or more independent observers. These experiments established that $10^{9} \mathrm{CFU}$ of strain CRMEN 44 is the MLD for 1-month-old BALB/c mice, with all mice that received the MLD dose dying within $48 \mathrm{~h}$ (see Fig. 1). This MLD was used in all the phage therapy experiments described in this study. A similar approach was used to establish the MLD for E. faecalis strain CRMEN 19 as $3 \times 10^{9} \mathrm{CFU}$. This strain was employed in experiments to determine the need for phage strains that demonstrate in vitro lytic activity against the specific bacterial strain involved in an infection.

Treatment with phage. The efficacy of phage therapy was evaluated in two separate experiments using the VRE bacteremia mouse model. The first examined the effect of phage dose on the ability of phage to rescue mice from VRE bacteremia. The second studied the effect on the outcome of delaying treatment for various periods. In the dose-ranging study, five groups of mice (five mice in each) were challenged by i.p. injection of the MLD of CRMEN 44. Each of these groups was treated with a single injection of phage ENB6, administered i.p. 45 min after the bacterial challenge at $3 \times 10^{9}, 3 \times 10^{8}, 3 \times 10^{6}, 3 \times 10^{4}$, and 0 PFU. As an additional control, a sixth group (two mice) was not challenged with bacteria, receiving only the injection of phage (at the highest dose). The state of the health of these animals was monitored for 20 days.

In the delayed-treatment study, treatment (a single injection of phage at the highest dose) was initiated at $5,8,12,16$, and $24 \mathrm{~h}$ after the bacterial challenge with the MLD of CRMEN 44. The state of the health of these animals was also monitored for 20 days.

Dependence of phage rescue on bacterial susceptibility. The capacities of phage strains to rescue bacteremic mice were correlated with their ability or inability to form plaques on the infecting bacterial strain. Mice were infected by i.p. injection with the MLD $\left(3 \times 10^{9} \mathrm{CFU}\right)$ of $E$. faecalis CRMEN 19. Five groups of mice (with 10 mice in each group) were compared. Two of the groups were treated with a single i.p. injection of $9 \times 10^{9}$ PFU of phage, ENB6 for one group and C33 for the other, administered 90 min after the i.p. injection of $3 \times$ $10^{9} \mathrm{CFU}$ of CRMEN 19. As a control, a third group of mice was injected i.p. with PBS instead of phage 90 min after the i.p. injection of $3 \times 10^{9}$ CFU of CRMEN 19. Two additional control groups were not challenged with bacteria but received a single i.p. injection of $9 \times 10^{9}$ PFU of phage strains ENB6 and C33, respectively, administered 90 min after an i.p. injection of PBS.

Effects of heat-inactivated phage. An experiment was performed to determine whether phage rescue of mice with VRE bacteremia requires phage that can grow on the bacterial host or whether phage rescue might be associated with a nonspecific immune activation response. A sample of phage ENB6 with a titer of $1.28 \times 10^{10} \mathrm{PFU} / \mathrm{ml}$ was heat inactivated by incubation at $80^{\circ} \mathrm{C}$. Phage that had been heated for a total of $20 \mathrm{~min}$, at which time no viable phage was detectable, was used to determine whether phage rescue of mice with VRE bacteremia requires functional phage or whether the rescue might be associated with nonspecific immune activation. The mice in this study were divided into three groups of 10 mice each. All of the mice were challenged by i.p. injection of the MLD of CRMEN 44. One group was treated with a single i.p. injection of $3 \times 10^{9} \mathrm{PFU}$ of phage ENB6 $45 \mathrm{~min}$ after the bacterial challenge. The second group was treated by i.p. injection with $3 \times 10^{9}$ PFU of heat-inactivated ENB6 phage particles 45 min after the bacterial challenge. The third group, a control, received a single injection of PBS instead of phage, administered i.p. $45 \mathrm{~min}$ after the bacterial challenge.

Measuring the immune response to the phage. At various time points following i.p. injection of phage ENB6 $\left(10^{10} \mathrm{PFU}\right)$, mouse serum was prepared, and indirect enzyme-linked immunosorbent assays were performed, as described by Harlow and Lane (8). For these assays, twofold serial dilutions of serum were prepared in 96-well polystyrene microtiter plates precoated with $1.7 \times 10^{9} \mathrm{PFU}$ of phage ENB6. Immunoglobulins were detected with goat anti-mouse immunoglobulin $\mathrm{M}$ (IgM)- or IgG-specific antibodies conjugated to peroxidase and tetramethylbenzadine (TMB) peroxidase substrate (Kirkegaard and Perry Laboratories, Gaithersburg, Md.).

Electron microscopy. Phage ENB6 was grown in a VSE host (ATCC 19950) by standard procedures and purified by cesium chloride density centrifugation. The preparation was then spread on an electron microscope grid and negatively stained with uranyl acetate.

\section{RESULTS}

Phage strain antibacterial activity. Of the phage strains isolated in these experiments, one (ENB6) was found to form plaques on $57 \%$ of the VRE clinical isolates, and it inhibited bacterial growth of an additional $22 \%$ of the strains, thus exhibiting an antibacterial effect against $79 \%$ of the strains in our collection. The inhibition of bacterial growth in strains that the phage could not form plaques on is most likely due to partial expression of the phage genome, sufficient for killing but not enough for phage production at a level necessary for plaque formation. As the phage preparations were purified by $\mathrm{CsCl}$, as mentioned earlier, it is unlikely that the observed inhibition of bacterial growth of the additional $22 \%$ of the strains was due to activity of lysins produced by bacterial lysis. 


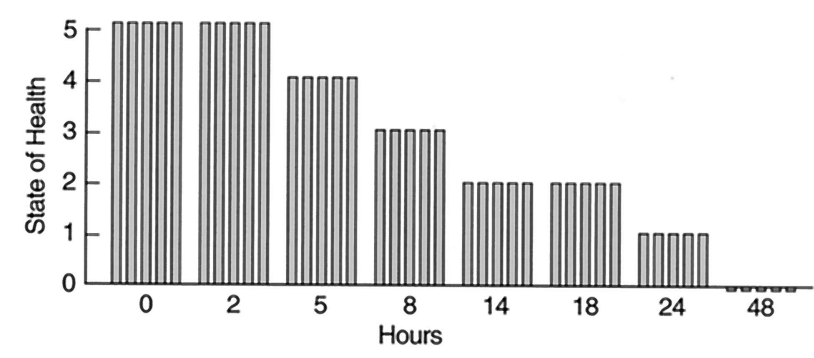

FIG. 1. Lethality of the MLD of VRE in this model. Mice were inoculated i.p. with $10^{9} \mathrm{CFU}$ of VRE strain CRMEN 44. A score of 5 indicates normal health, while 0 indicates death (see the text for a full description of the scale). Each bar indicates the state of health of a single BALB/c mouse. All animals were dead within $48 \mathrm{~h}$.

Lethality of VRE bacteremia. As seen in Fig. 1, all mice inoculated i.p. with the MLD $\left(10^{9} \mathrm{CFU}\right)$ of the clinical isolate VRE strain CRMEN 44 died within $48 \mathrm{~h}$.

Ability of the phage preparation to rescue mice from VRE bacteremia. A single dose of phage ENB6 was administered i.p. $45 \mathrm{~min}$ after the challenge with the MLD of bacteria. By $24 \mathrm{~h}$ a dose effect on the state of health of the infected animals was clearly visible. At higher doses of phage, which achieved multiplicities of infection of 0.3 and $3.0,100 \%$ of the animals survived, and only minimal signs of illness (mild lethargy) were seen (in the first $24 \mathrm{~h}$ ). As the phage dose decreased, resulting in lower multiplicities of infection ( 0.003 and 0.03 ), the animals became critically ill, with survival rates of 40 and $60 \%$, respec- tively, at day 6 and beyond (Fig. 2). The two-by-five chi square comparing the survival rates at day 6 was statistically significant: chi square $=13.8$, df $=4, P<0.008$. In addition, the Armitage test for the trend in survival rates indicates a statistically significant decreasing trend in survival rates $(P<$ $0.0005)$ (3). All of the mice that were alive and healthy at day 6 remained so for an additional 20 days, at which point the experiment was terminated.

Rescue is associated with a significant decrease in bacterial titer. In a similar experiment, blood was obtained by cardiac puncture during a rescue experiment, in order to compare bacterial titers from two groups of 10 mice, $20 \mathrm{~h}$ after i.p. inoculation with $3 \times 10^{9} \mathrm{CFU}$ of CRMEN 44 . Forty-five minutes after the bacterial inoculation of the first group, $300 \mu \mathrm{l}$ of PBS was injected i.p. as a control. The mean bacterial titer in the blood $20 \mathrm{~h}$ after bacterial inoculation for this group of mice was $2.03 \times 10^{7} \pm 1.10 \times 10^{7} \mathrm{CFU} / \mathrm{ml}$ (mean \pm standard error). Forty-five minutes after bacterial inoculation of the second group, the mice were injected i.p. with $9 \times 10^{9} \mathrm{PFU}$ of phage ENB6. The mean bacterial titer in the blood $20 \mathrm{~h}$ after bacterial inoculation for this group was $8.74 \times 10^{4} \pm 6.03 \times 10^{4}$ $\mathrm{CFU} / \mathrm{ml}$. Phage therapy thus resulted in a 200 -fold decrease (compared to the control group) in blood bacterial titers at $20 \mathrm{~h}$. This reduction in bacterial titers was reflected in the lower morbidity and mortality observed in the treated group. Whereas at the 20-h time point the control group had a median health score of 2 (severe illness), with scores ranging from 0 (one death) to 4 , in contrast the phage-treated group had a
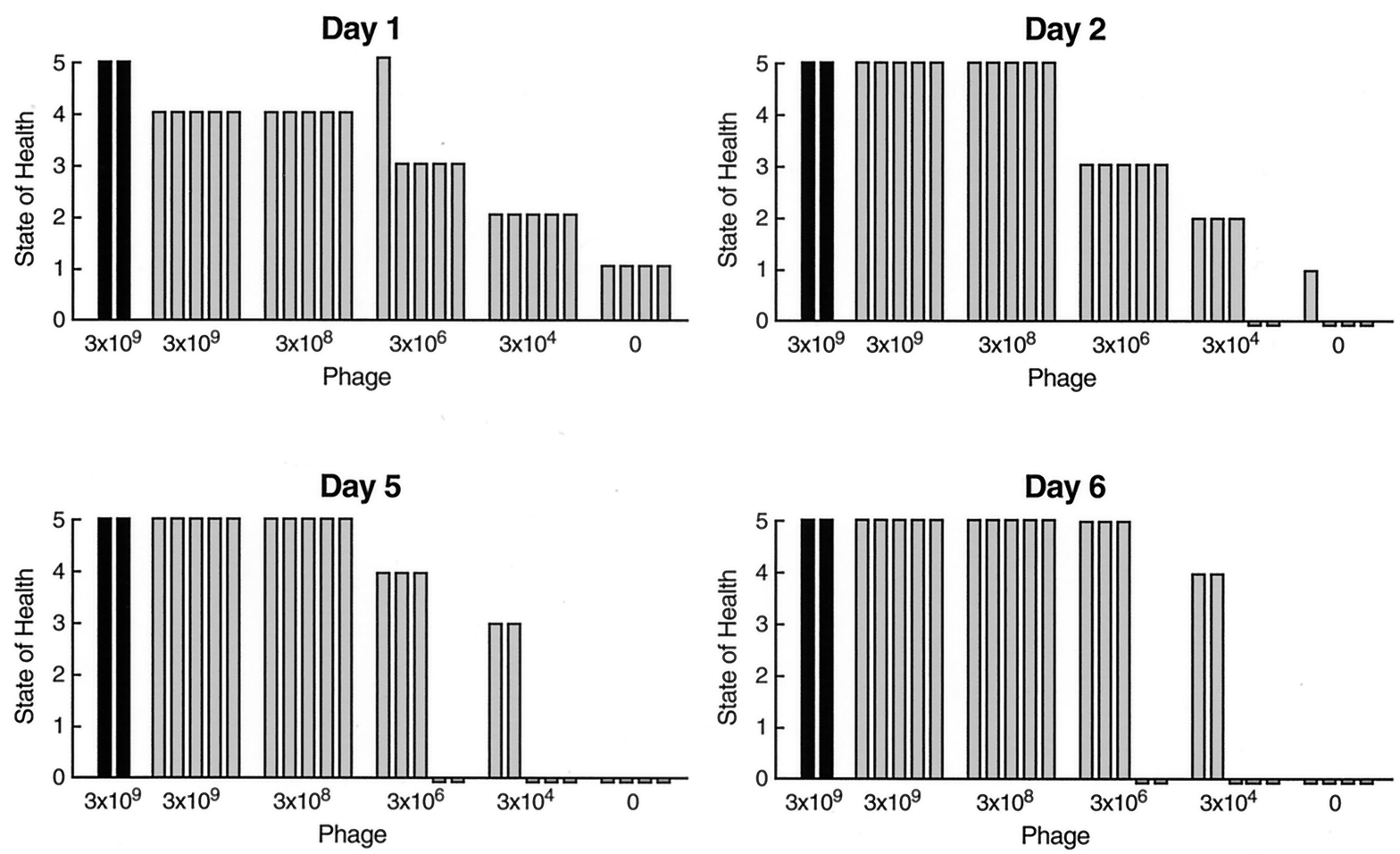

FIG. 2. Dose effect of phage ENB6 in rescuing mice from lethal VRE bacteremia. The mice were scored for their state of health as described in the text. Each bar indicates the state of health of mice after i.p. administration of the MLD of VRE followed by a single dose of phage at the indicated concentration $45 \mathrm{~min}$ after the bacterial challenge. The group on the far right (four mice) was an untreated control, given SM buffer i.p. instead of phage. The group on the far left (two animals) was a phage control, injected only with phage (at the high dose) and not infected with bacteria, in order to determine if the phage preparation itself produces adverse effects in mice. 

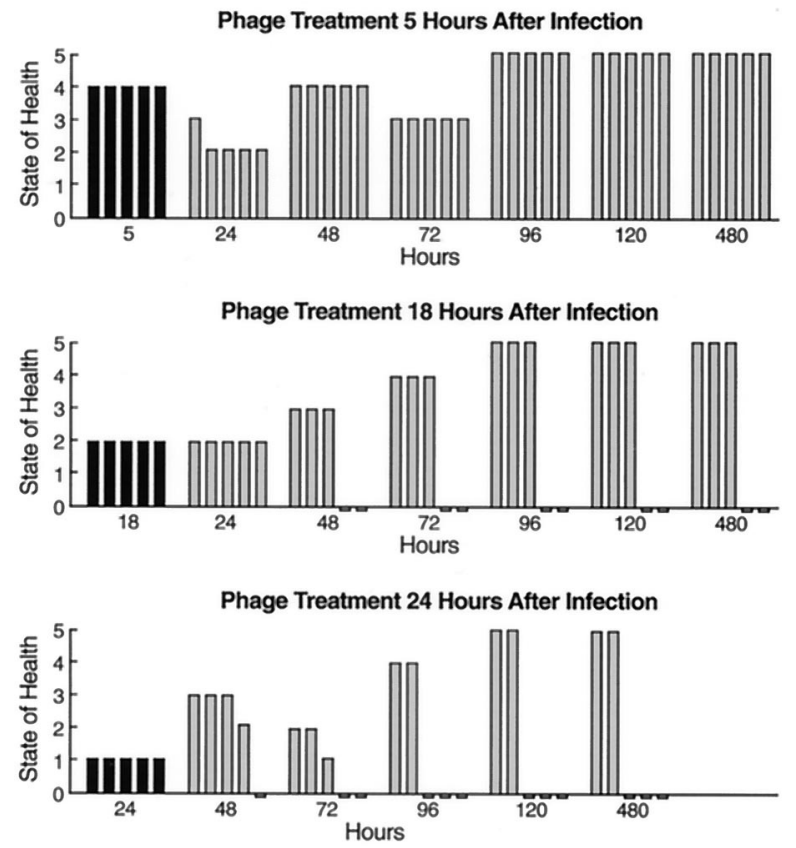

FIG. 3. Delayed phage treatment of bacteremic mice. A single i.p. injection of the highest dose of the phage preparation $\left(3 \times 10^{9} \mathrm{PFU}\right)$ was administered to bacteremic mice at the indicated times after bacterial challenge. The mice were scored for their state of health as described in the text. Black and gray bars show their condition at the time of phage treatment and following phage treatment, respectively. When treatment was delayed for $24 \mathrm{~h}$, all the animals were moribund, but approximately half of them not only survived but also went on to recover completely as a result of the single dose of phage.

median health score of 3 (moderate illness), with no deaths and scores ranging from 2 to 5 . In both groups there was a correlation between the state of health of the mice and the concentration of bacteria in the blood. Mice with bacterial titers of $10^{7} \mathrm{CFU} / \mathrm{ml}$ or more had a score of 1 (moribund condition), while titers in the range of $10^{6}$ to $10^{4} \mathrm{CFU} / \mathrm{ml}$ resulted in a score of 2. Mice with $10^{4}$ to $10^{3} \mathrm{CFU}$ of bacte$\mathrm{ria} / \mathrm{ml}$ in their blood displayed a score of 3 , and mice with titers of $10^{2} \mathrm{CFU} / \mathrm{ml}$ or less had scores of 4 to 5 (mild illness or no signs of illness, respectively). This relationship between bacterial concentration and the state of the health of the mice had an overall Pearson correlation of -0.931 and a Spearman rank correlation of -0.921 .

Effect of delay in treatment on the ability of the phage preparation to rescue mice from VRE bacteremia. In this experiment, the MLD of VRE strain CRMEN 44 was injected i.p. to induce fatal bacteremia. At various intervals thereafter, ranging from 2 to $24 \mathrm{~h}$, the mice received a single i.p. injection of the high dose $\left(3 \times 10^{9} \mathrm{PFU}\right)$ of phage ENB6. The results of treatment at the time points 5,18 , and $24 \mathrm{~h}$ time points are illustrated in Fig. 3. The state of health of these mice was monitored for 20 days following bacterial infection. The experiments demonstrate that (i) a single injection of phage can rescue $100 \%$ of the animals, even when treatment is delayed until $5 \mathrm{~h}$ after lethal bacterial challenge, and (ii) if treatment is delayed beyond that point, morbidity increases and mortality begins to appear. However, even with delays of 18 and $24 \mathrm{~h}$, at which point all the mice are moribund, approximately $50 \%$ of the animals are rescued and go on to recover completely. Using linear regression, where survival rates were compared with the time of survival following the initial bacterial infection, we found that the upper $95 \%$ confidence limit on the window of time for treatment after infection may possibly be extended to $7 \mathrm{~h}$ and still maintain $100 \%$ survival (6).

Dependence of phage rescue on bacterial susceptibility. We compared the capacities of two different phage strains to rescue mice that were made bacteremic by i.p. injection with $3 \times$ $10^{9} \mathrm{CFU}$ of $E$. faecalis CRMEN 19. This bacterial strain is resistant in vitro to the phage ENB6 at the concentrations used in this study, but it is susceptible to phage C33, a strain with a wider host range than ENB6. ENB6 displayed no plaque-forming ability on CRMEN 19 at the concentrations used in this experiment, while another phage strain with a wider bacterial host range, C33, does form plaques on this bacterial strain. Treatment of bacteremic animals with phage C33 (group D) resulted in a $100 \%$ survival rate (similar to that observed in the healthy mice injected with phage [groups A and B]) 20 days after infection, compared with a survival rate of $50 \%$ in the untreated control mice (group E) (Fig. 4). Post hoc testing using Fisher's exact test indicates that group E (50\% survival) is statistically significantly different from each of the $100 \%$ survival groups (groups $\mathrm{A}, \mathrm{B}$, and $\mathrm{D} ; P<0.03$ ).

In contrast, treatment with phage ENB6 resulted in a 20\% survival rate in group $\mathrm{C}$, which was lower than that in the untreated controls. Post hoc testing using Fisher's exact test indicates that group C (20\% survival) is statistically significantly different from each of the $100 \%$ survival groups (groups $\mathrm{A}, \mathrm{B}$, and $\mathrm{D} ; P<0.0007)$. The two-by-five chi square comparing the survival rates at day 20 was statistically significant: chi square $=28.7, \mathrm{df}=4, P<0.00001$. The comparison of group C (20\% survival) to group E (50\%) had a Fisher $P$ value of $<0.2$.

The lower survival rate observed in mice with $E$. faecalis CRMEN 19 bacteremia treated with ENB6 phage was dependent on the amount of phage used. In additional experiments (not illustrated), CRMEN 19 bacteremic mice treated with the phage ENB6 preparation at $9 \times 10^{8}, 9 \times 10^{7}$, and $9 \times 10^{6} \mathrm{PFU}$ had 6-day survival rates of 30,30 , and $40 \%$, respectively. It should be noted that phage preparations of ENB6 and C33 were purified to the same degree by $\mathrm{CsCl}$ density centrifugation followed by dialysis with PBS. No adverse effects were noted when healthy mice were inoculated i.p. with the highest dose, $9 \times 10^{9} \mathrm{PFU}$, of either phage, as illustrated by the data for groups A and B in Fig. 4.

Effects of heat-inactivated phage. An experiment was performed to determine whether phage rescue of mice with VRE bacteremia requires functional phage or whether phage rescue might be associated with a nonspecific immune activation response. Heat-inactivated phage were used to test whether a functional phage and not a nonspecific immune response is responsible for the rescue of mice inoculated with a lethal dose of VRE. Heating at $80^{\circ} \mathrm{C}$ for $5 \mathrm{~min}$ decreased the phage titer by 1,000 -fold, and no viable phage was detected after heating for 15 or $20 \mathrm{~min}$. As illustrated in Fig. 5, only mice inoculated with plaque-forming phage had enhanced survival, with $80 \%$ survival at 4 days, while only $10 \%$ of the PBS control mice and $10 \%$ of the mice injected with heat-inactivated phage survived. 

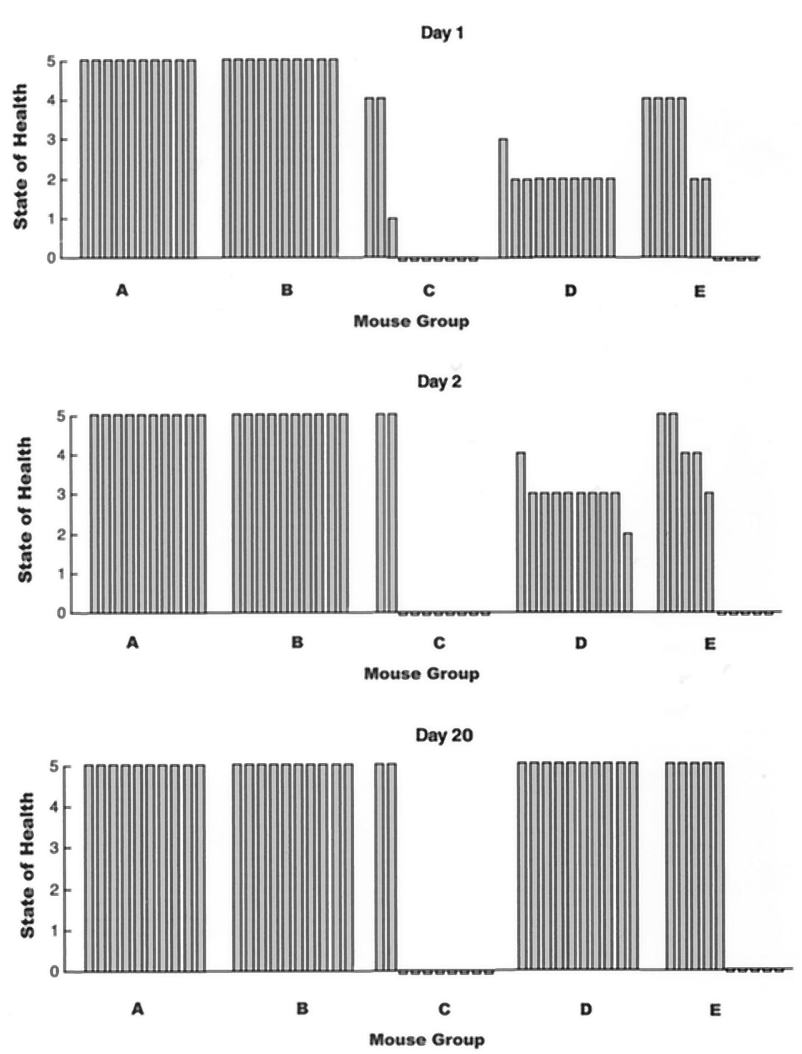

FIG. 4. Comparison of phage strains ENB6 and C33, which cannot and can form plaques in vitro on VRE strain CRMEN 19, respectively, with their ability to rescue mice that are bacteremic with CRMEN 19. Groups A and B were healthy mice treated with a single i.p. injection of $9 \times 10^{9} \mathrm{PFU}$ of ENB6 and C33, respectively. Groups C and D were treated with $9 \times 10^{9} \mathrm{PFU}$ of ENB6 and C33, respectively, 90 min after bacterial infection. Group E was an untreated control, injected i.p. with PBS buffer $90 \mathrm{~min}$ after the bacterial challenge instead of phage. Treatment with phage strain C33 resulted in 100\% recovery from bacteremia. This recovery rate is significantly better than the survival rates of 20 and $50 \%$ for the infected mice treated with ENB6 and with PBS, respectively.

These results were statistically significant. The chi square with 2 degrees of freedom is $14.7(P<0.0006)$. These statistical results are the same for the observations made on days 2 and 3 as well, since the data pattern is the same at days 2, 3, and 4 . The animals were monitored for an additional 20 days, and no changes in their state of health were noted.

Immune response to phage ENB6. After the third in a series of five monthly injections of phage ENB6, titers of IgG and IgM raised against the phage increased above background 3,800-fold and 5-fold, respectively (Fig. 6). IgG levels did not change substantially after the third injection. No anaphylactic reactions, changes in core body temperature, or other adverse events were observed in the mice over the course of these multiple injections of phage.

Electron microscopy. As seen in Fig. 7, phage ENB6 has a moderately elongated head, $99.7 \mathrm{~nm}$ long and $84.4 \mathrm{~nm}$ wide, and a contractile tail (199.1 nm long). The phage is of the A2 morphotype (1), as observed by negative staining with uranyl acetate.

\section{DISCUSSION}

VRE bacteremia is often lethal for immunocompromised patients. The bacteria gain entry to the bloodstream from various sites, e.g., by translocating from (otherwise asymptomatic) intestinal colonization, by entry from an infected urinary tract or from infected skin, and as a result of intra-abdominal abscesses. We developed an animal model to determine the potential of phage to cure such VRE bacteremias.

The results obtained in the present experiments are encouraging: a single i.p. injection of either of the two higher dosages of phage ENB6 used in this study $\left(10^{9}\right.$ and $\left.10^{8} \mathrm{PFU}\right)$ rescued $100 \%$ of VRE bacteremic mice, even if treatment was delayed up to $5 \mathrm{~h}$. Moreover, even when phage treatment (with the highest dose) was delayed until all animals were moribund, approximately half the animals were rescued and went on to recover completely. The ability of phage to rescue bacteremic animals was a phage function and not a nonspecific immune response activation, since rescue was observed with heat-inactivated phage. Furthermore, by comparing phage strains (C33 and ENB6) that can and cannot form plaques in vitro on $E$. faecalis CRMEN 19, respectively, it was found that rescue of CRMEN 19 bacteremic mice was limited to C33, which displayed in vitro lytic activity against that bacterial host. The lower survival rate observed in CRMEN 19 bacteremic mice treated with phage ENB6 indicates that stressed animals are more sensitive to various factors, in this case either the phage itself or trace amounts of endo- and exotoxins present in the phage preparations. Healthy animals displayed no apparent reaction to these factors, as shown by the lack of any adverse effect in the control group inoculated with a high dose of the ENB6 phage preparation (Fig. 4).

Each of the above experiments independently demonstrates the requirement for active phage, that is, phage that are capable of growing in and lysing the infecting bacterial strain, for the rescue of animals that are bacteremic with that bacterial strain. This conclusion was further strengthened by observations concerning the fate of the bacteria during infections. In these experiments we observed a 200 -fold decrease in blood bacterial titers in mice treated with phage therapy compared with blood bacterial titers from the PBS-treated control mice $20 \mathrm{~h}$ after initiation of the experiment. In addition, we found that the blood bacterial titers correlated with the morbidity and mortality observed in these groups.

The present experiments extend previous studies from our laboratory, in which a single i.p. injection of the appropriate phage rescued mice with bacteremic due to $E$. coli or $S$. enterica serovar Typhimurium (11). These experiments also tend to corroborate a study by Smith and Huggins in which mice were inoculated with lethal intramuscular or intracerebral injections of $E$. coli and a single injection of a coliphage preparation rescued the animals (15). Interestingly, that study demonstrated that a single intramuscular dose of phage was more effective than multiple intramuscular injections of antibiotics, such as tetracycline, ampicillin, chloramphenicol, or trimethoprim plus sulfisoxazole. Levin and Bull extended the Smith and Huggins study in a population dynamics model which demonstrated that it was the exponential growth in numbers of phage particles that enabled a single injection of phage to be superior to multiple injections of antibiotics (10). In still 

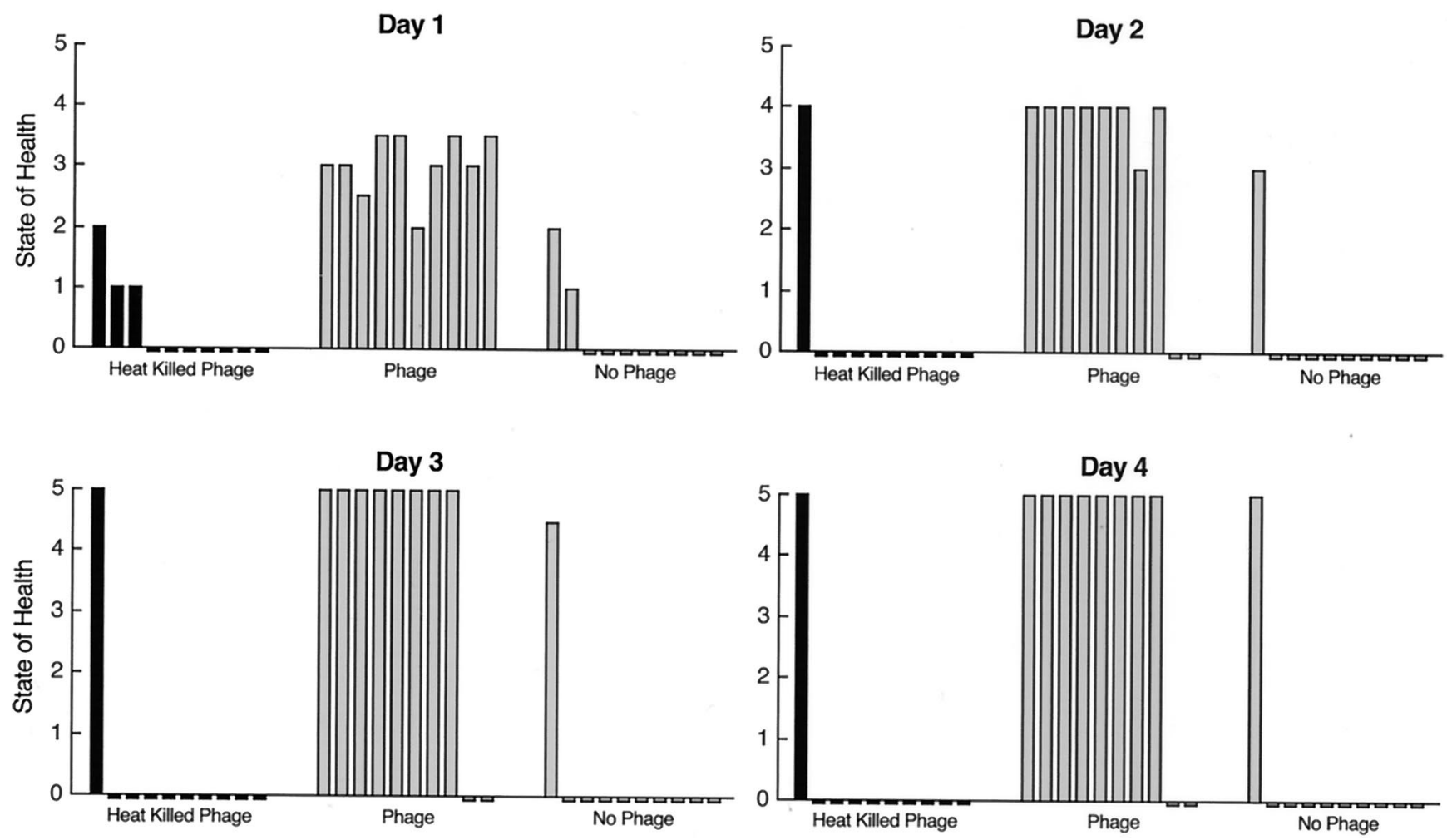

FIG. 5. Comparison of the ability of phage and nonfunctional heat-inactivated phage to rescue bacteremic mice. One group of 10 mice was treated with functional (plaque-forming) phage, while the other two groups were treated either with no phage or heat-inactivated phage. Eighty percent of the bacteremic mice treated with functional phage survived, while only $10 \%$ of the mice in each of the other two groups survived. The animals were monitored for 20 more days, and no changes in their state of health were noted.

other studies, preparations of the appropriate phage have been able to protect mice and guinea pigs against systemic infections with strains of Pseudomonas aeruginosa and Acinetobacter baumanii and have been able to inhibit the rejection of skin grafts caused by $P$. aeruginosa infections $(16,17)$.

Although phages were discovered nearly a century ago, Western medicine's interest in them as therapeutic agents was relatively short-lived, in part because of the eventual discovery and immediate success of antibiotics and in part because of the

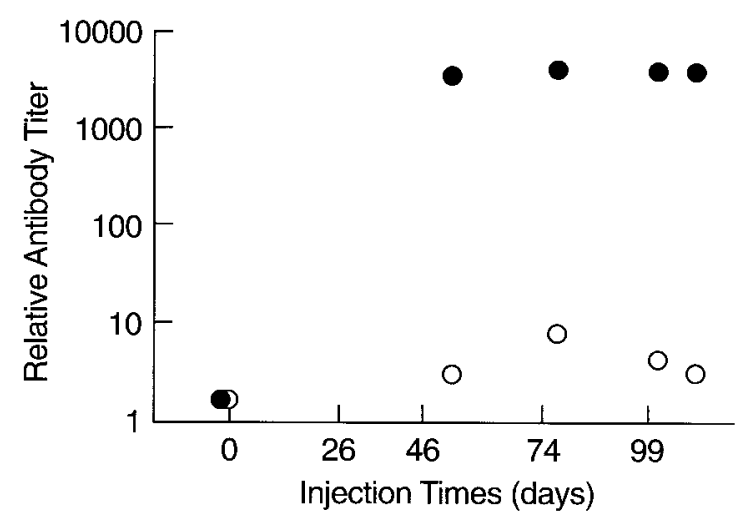

FIG. 6. Antibody responses in mice to repeated injections of phage ENB6. Phage were injected i.p. at the indicated time points. The resulting titers of anti-ENB6 $\operatorname{IgG}(\bullet)$ and $\operatorname{IgM}(\bigcirc)$ antibodies are indicated. highly empirical and counterproductive approach that had been used by phage practitioners in the early era. In the modern era (1980s and 1990s), some rigorously controlled animal experiments have been conducted (15-17), but the clinical

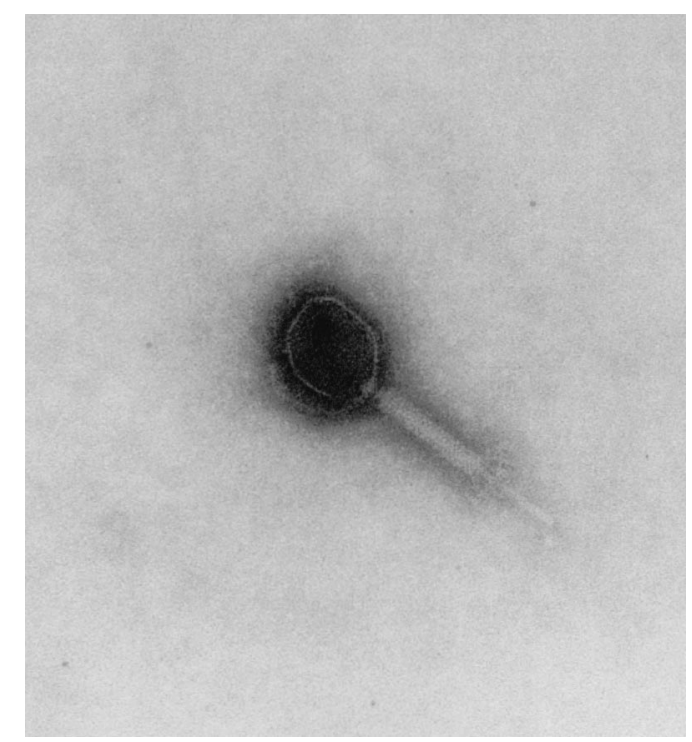

FIG. 7. Electron micrograph of phage ENB6 negatively stained with uranyl acetate. The phage head is elongated, with a length of 99.7 $\mathrm{nm}$ and width of $84.4 \mathrm{~nm}$. The contractile tail is $199.1 \mathrm{~nm}$ in length. 
reports in this same era have been of an anecdotal nature rather than describing controlled studies (2). The experiments in the present study represent solutions to many of the problems that hindered the prior applications of phage therapy. For example, the relatively narrow host range of most phages, which caused many of the early attempts at phage therapy to fail, can be overcome by isolating phages that have a broad host range within the species being targeted. For example, phage ENB6 forms plaques on more than $50 \%$ of the VRE isolates tested and inhibits growth of an additional $20 \%$, thus providing an antibacterial effect against more than $70 \%$ of approximately 100 clinical isolates of VRE taken from diverse geographic regions in the United States. Reduction of harmful bacterial debris (containing endo- and exotoxins) often present in phage preparations was achieved by using cesium chloride density centrifugation to purify the phage preparations.

While phage ENB6 invokes an immune response in mice, the antibodies raised over the course of several months of repeat injections were not associated with anaphylaxis or other adverse reactions. In any case, these experiments were designed as a model for acute human infections, in which antibiotics are no longer effective and a single course of phage treatment might rescue the patient. If phage are to be employed repeatedly, e.g., for chronic infections, it may be possible to use selection and phage display to derive phage variants that are less prone to induce an immune response.

With the emergence of antibiotic-resistant bacteria, such as VRE and methicillin-resistant Staphylococcus aureus, there is a need to explore the potential therapeutic applications of bacteriophages. That exploration is facilitated by knowledge gained during the development of the science of molecular biology about the mechanisms of phage interactions with bacteria. This knowledge, coupled with rigorously controlled animal models and clinical trials, should permit the rational development of phage therapy. In this regard, the inocula of bacteria used in the animal experiments described in this study are higher than those normally encountered in clinical situations. However, the ability of phage to rescue animals infected with these high titers of bacteria is especially convincing of the potential of phage as an antibacterial therapeutic agent. Given the encouraging results of these initial experiments, it may be useful to develop more sophisticated experiments that portray normal clinical situations.

The potential of phage therapy has been the subject of several recent reviews $(2,4,13,18)$, and the present study reinforces the view that this potential is worth exploring. We focused our efforts on VRE because we anticipated that positive results would demonstrate the potential of this form of therapy in situations where few alternatives are available today.

\section{ACKNOWLEDGMENTS}

We thank John Bartko for help with the statistical analysis of the data and Desiree von Kollmar for help in preparing the figures.

\section{REFERENCES}

1. Ackermann, H.-W., and M. S. Dubow. 1987. Viruses of prokaryotes, p. 13-28. CRC Press, Inc., Boca Raton, Fla.

2. Alisky, J., K. Iczkowski, A. Rapoport, and N. Troitsky. 1998. Bacteriophages show promise as antimicrobial agents. J. Infect. 36:5-15.

3. Armitage, P. 1955. Tests for linear trends in proportions and frequencies. Biometrics 2:375-386.

4. Carlton, R. M. 1999. Phage therapy: past history and future prospects. Arch. Immunol. Ther. Exp. 47:267-274.

5. Cimons, M. 1999. FDA approves the antibiotic Synercid for limited clinical uses. ASM News 65:800-801.

6. Draper, N. R., and H. Smith. 1966. Applied regression analysis, 2nd ed., p. 47-49. John Wiley \& Sons, Inc., New York, N.Y.

7. Geier, M. R., M. E. Trigg, and C. R. Merril. 1973. The fate of bacteriophage lambda in non-immune germfree mice. Nature 246:221-223.

8. Harlow, E., and D. Lane. 1999. Using antibodies, a laboratory manual. Cold Spring Harbor Laboratory Press, Cold Spring Harbor, N.Y.

9. Leclercq, R., E. Derlot, J. Duval, and P. Courvalin. 1998. Plasmid-mediated resistance to vancomycin and teicoplanin in Enterococcus faecium. N. Engl. J. Med. 319:157-161.

10. Levin, B., and J. J. Bull. 1996. Phage therapy revisited: the population biology of a bacterial infection and its treatment with bacteriophage and antibiotics. Am. Nat. 147:881-898.

11. Merril, C. R., B. Biswas, R. Carlton, N. C. Jensen, G. J. Creed, S. Zullo, and S. Adhya. 1996. Long-circulating bacteriophage as antibacterial agents. Proc. Natl. Acad. Sci. USA 93:3188-3192.

12. Murray, B. E. 2000. Vancomycin-resistant enterococcal infections. N. Engl. J. Med. 342:710-721.

13. Pirisi, A. 2000. Phage therapy—advantages over antibiotics? Lancet 356: 1418 .

14. Sambrook, J., E. F. Fritsch, and T. Maniatis. 1989. Molecular cloning: a laboratory manual, 2nd ed., vol. 1, p. 66-79. Cold Spring Harbor Laboratory Press, Cold Spring Harbor, N.Y.

15. Smith, H. W., and M. B. Huggins. 1982. Successful treatment of experimental Escherichia coli infections in mice using phage: its general superiority over antibiotics. J. Gen. Microbiol. 128:307-318.

16. Soothill, J. S. 1992. Treatment of experimental infections of mice with bacteriophages. J. Med. Microbiol. 37:258-261.

17. Soothill, J. S. 1994. Bacteriophage prevents destruction of skin grafts by Pseudomonas aeruginosa. Burns 20:209-211.

18. Soothill, J. S., and P. A. Barrow. 1997. Bacteriophage therapy and prophylaxis: rediscovery and renewed assessment of potential. Trends Microbiol. 5:268-271.

19. Uttley, A. H. C., C. H. Collins, J. Naidoo, and R. C. George. 1988. Vancomycin-resistant enterococci. Lancet i:57-58.

Editor: J. T. Barbieri 